\title{
Recurrent right loin abscess: an atypical presentation of chronic appendicitis
}

\author{
Prabhakaran $\mathrm{S}^{*}$, Li R and Chandra R \\ Department of Specialist General Surgery, The Royal Melbourne Hospital, Parkville, VIC, Australia
}

\begin{abstract}
We report the unusual case of a 71-year-old female who had recurrent presentations over three years with right loin abscess appearing to originate from a retroperitoneal collection. She was initially diagnosed with a right psoas abscess of unknown aetiology and was managed with open surgical drainage of this collection. However, further investigations after recurrent admissions raised the suspicion of appendicitis as the underlying pathology. Despite two unremarkable colonoscopies, she proceeded to undergo diagnostic laparoscopy and appendicectomy, with histopathology ultimately showing chronic appendicitis. She made an unremarkable recovery.
\end{abstract}

\section{Case report}

We report the unusual case of a 71-year-old female who had recurrent presentations over a period of three years with a right loin abscess appearing to originate from a retroperitoneal collection. She was initially diagnosed with a psoas abscess of uncertain aetiology and was managed with open surgical drainage of this collection. However, further investigations after recurrent admissions raised the suspicion of appendicitis. At laparoscopy, the appendix was identified as the source of her collection and she underwent a laparoscopic appendicectomy with an uneventful recovery.

The patient initially presented with three weeks of right loin erythema, a palpable fluctuant mass and malaise. She denied fevers, bowel symptoms, urinary symptoms, night sweats, anorexia or weight loss. Her past medical history included hypertension, asthma and previous Hepatitis B infection; she was an immigrant from South East Asia.

$\mathrm{CT}$ abdomen/pelvis revealed a large right psoas abscess measuring $12 \times 10 \times 12 \mathrm{~cm}$ (Figure 1) which extended through the right abdominal wall into subcutaneous tissue. Ultrasound-guided aspiration suggested that this was likely an abscess, as the aspirate was negative for malignancy

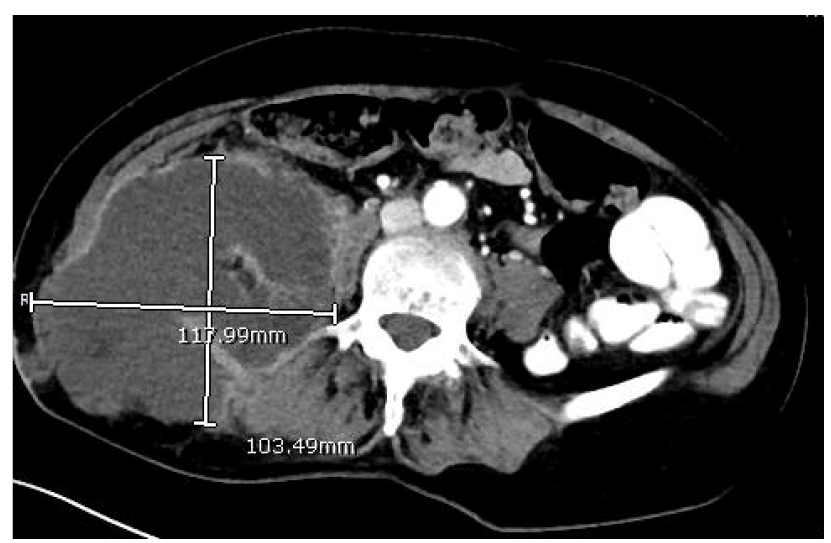

Figure 1. $\mathrm{CT}$ at initial presentation, demonstrating a large right retroperitoneal abscess extending beyond the right psoas muscles into the subcutaneous tissues of the right loin area and tuberculosis. The patient underwent incision and drainage of this in theatre with drainage of approximately $1200 \mathrm{ml}$ of fluid. This cultured Citrobacter koseri and Bacteroides fragilis. She had a percutaneous drain left in situ and received intravenous antibiotics (initially ceftriaxone and metronidazole, then broadened to meropenem, then to piperacillin and tazobactam) for two weeks in total, until repeat imaging showed a reduction in the size of the collection to $2 \times 6 \times 3 \mathrm{~cm}$. The drain tube was then removed, and she was discharged with a two-week course of oral amoxicillin/clavulanic acid. An outpatient colonoscopy performed five months post discharge was unremarkable.

She represented two years later with ongoing abdominal pain of one-year duration, associated with vomiting and fevers. She was noted again to have cellulitis, with CT again demonstrating a $6 \times 2 \mathrm{~cm}$ abscess adjacent to the tip of the appendix (Figures 2,3). She underwent CTguided drainage, with fluid culturing Escherichia coli, Citrobacter koseri, Klebsiella pneumoniae, Bacteroides fragilis and Fusobacterium varium. On specialist infectious diseases advice, she was treated with intravenous ceftriaxone and metronidazole for two weeks in total, at which point she demonstrated marked clinical and biochemical improvement. She was then discharged home with oral amoxicillin/ clavulanic acid for two weeks.

She represented two months later with ongoing right loin pain associated with cellulitis. She had no fevers, but CT showed a $6 \times 3 \times 3 \mathrm{~cm}$ subcutaneous fluid collection extending into the right iliopsoas muscle. She underwent ultrasound guided drainage. Fluid cultured Provindecia rettgarei and Citrobacter freundii. She was treated with intravenous antibiotics (initially ceftriaxone for two days, meropenem for three days, then ertapenem for four weeks), and changed to oral antibiotics (amoxicillin/clavulanic acid and ciprofloxacin) for four weeks when improved.

${ }^{*}$ Correspondence to: Sowmya Prabhakaran, Department of Specialist General Surgery, The Royal Melbourne Hospital, Parkville, VIC, Australia, E-mail: Sowmya.Prabhakaran@mh.org.au

Key words: abscess of the abdominal wall, appendicitis

Received: April 16, 2018; Accepted: May 14, 2018; Published: : May 17, 2018 


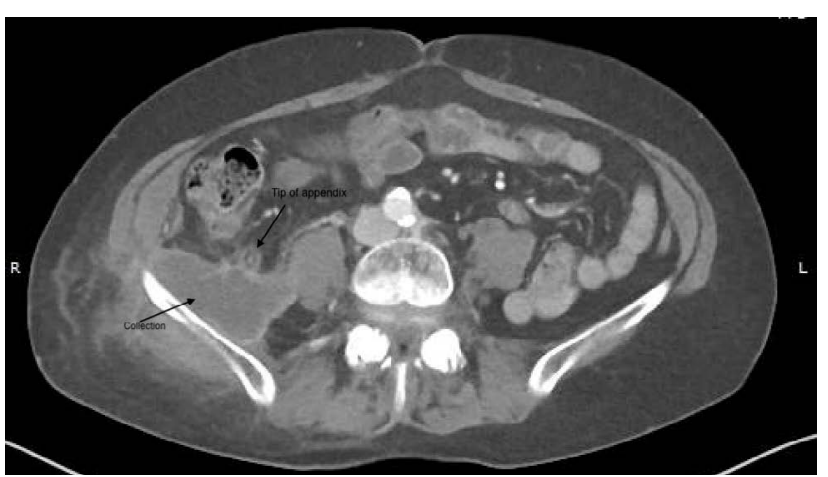

Figure 2. CT from the second presentation, showing the tip of appendix adjacent to the retroperitoneal collection

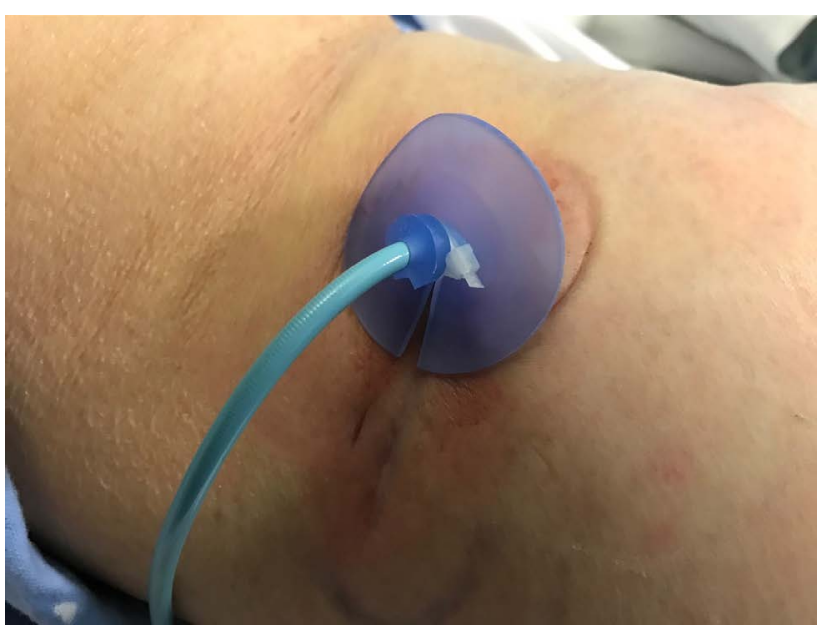

Figure 3. Clinical photograph, showing the right loin with percutaneous drain into abscess and surrounding cellulitis

Given that this was the third presentation of recurrent right loin collection from an iliopsoas abscess, and the CT demonstrating the proximity of the tip of the appendix, the patient was further investigated. A repeat outpatient colonoscopy four months later showed a normal caecum, with the base of the appendix appearing normal.

She was planned for diagnostic laparoscopy but represented to hospital prior to her planned surgery date with the same symptoms, with a CT abdomen/pelvis showing $6 \times 2 \mathrm{~cm}$ collection over the iliopsoas. Ultrasound guided drainage was performed, and cultures grew Escherichia coli, Streptococcus mitis and Haemophilus parainfluenzae. She had a percutaneous drain left in situ and remained on oral antibiotics until diagnostic laparoscopy.

She ultimately underwent a diagnostic laparoscopy a month later, which showed a normal caecum and a retrocaecal appendix, which was firmly adherent to the lateral paracolic side wall with granulation tissue and connecting to a retroperitoneal cavity. This was presumed to be the aetiology of her recurrent iliopsoas abscess. The appendix was accordingly removed, and a thorough lavage of the cavity was performed. Formal histopathology confirmed chronic appendicitis. She had an unremarkable postoperative course, and outpatient follow-up; there has been no recurrence of the abscess after one year of follow-up.

\section{Discussion}

This case describes an unusual manifestation of a common pathology, with chronic appendicitis presenting as a right loin abscess with associated cellulitis. Appendicitis, in particular retrocaecal appendicitis, is a well-recognized cause of psoas abscesses; however chronic macroscopic abscess formation of the loin is rare and has not been described in the literature. The diagnosis of appendicitis should be considered in patients with no other clear cause of right loin abscess or cellulitis associated with a psoas abscess.

There are few cases in the literature of appendicitis presenting as soft tissue infections of the loin or abdominal wall. Van Hejillak et al. described a case of a retroperitoneal appendicular abscess presenting as right thigh cellulitis in a 73-year-old woman. Similar to our case, this patient did not have any abdominal symptoms to suggest an intra-abdominal pathology. However, in this case the CT clearly demonstrated a perforated retrocaecal appendix as the cause [1]. Fanning et al. published a case of perforated acute appendicitis causing a right lumbar abscess in a 66-year-old male who was a type2 diabetic; similarly, Kawashima et al. reported a 78-year-old female with appendicitis causing right gluteal cellulitis $[2,3]$. In each case, CT was critical in achieving the diagnosis and correct management of the pathology. However, this is the first reported case of chronic appendicitis manifesting as a right loin abscess with associated cellulitis causing morbidity over many years. Moreover, in our patient, the appendix was not ruptured at operation with histopathology showing chronic appendicitis.

There are several important learning points from our case. First, cultures from her original swabs grew atypical organisms not usually associated with skin. This finding is usually an indication of a deeper abdominal infection. Moreover, the patient had two normal colonoscopies where the appendiceal base was visualized and macroscopically normal. If appendicitis cannot be ruled out with axial imaging, it may be prudent to proceed to diagnostic laparoscopy. In this case, laparoscopy was both diagnostic and therapeutic. It also highlights the need to consider common pathologies as a possible aetiology of uncommon presentations.

This case highlights the general principle that recurrent episodes of infection should prompt consideration of unusual causes, especially from organs within anatomical proximity. Imaging should be considered in unexplained recurrent skin and soft tissue infections to help delineate a deeper source of infection. Finally, in the context of ongoing infections in the right lower quadrant and areas of proximity, appendicitis should always remain an important differential. Diagnostic laparoscopy remains a clinically useful tool for both diagnosis and treatment in this clinical scenario.

\section{References}

1. van Hulsteijn LT, Mieog JS, Zwartbol MH, Merkus JW, van Nieuwkoop C (2017) Appendicitis Presenting As Cellulitis of the Right Leg. J Emerg Med 52: e1-e3. [Crossref]

2. Fanning DM, Barry M, O'Brien GC, Leahy AL (2007) Perforated retrocaecal appendix presenting as right lumbar abscess. Ir J Med Sci 176:125-128. [Crossref]

3. Kawashima M, Hashimoto Y, Sudo T, Uemura K, Nakashima A, et al. (2012) A rare diagnostic challenge in acute appendicitis: a case report. Hiroshima J Med Sci 61: 1921. [Crossref]

Copyright: (C2018 Prabhakaran S. This is an open-access article distributed under the terms of the Creative Commons Attribution License, which permits unrestricted use, distribution, and reproduction in any medium, provided the original author and source are credited. 